\title{
The $h$-vector of a Gorenstein toric ring of a compressed polytope
}

\author{
Hidefumi Ohsugi \\ Department of Mathematics \\ Faculty of Science \\ Rikkyo University \\ Toshima, Tokyo 171-8501, Japan \\ ohsugi@rkmath.rikkyo.ac.jp \\ Takayuki Hibi \\ Department of Pure and Applied Mathematics \\ Graduate School of Information Science and Technology \\ Osaka University \\ Toyonaka, Osaka 560-0043, Japan \\ hibi@math.sci.osaka-u.ac.jp
}

Submitted: May 8, 2005; Accepted: August 9, 2005; Published: October 1, 2005

Mathematics Subject Classifications: 52B20, $13 \mathrm{H} 10$

Dedicated to Richard P. Stanley on the occasion of his 60th birthday

\begin{abstract}
A compressed polytope is an integral convex polytope all of whose pulling triangulations are unimodular. A $(q-1)$-simplex $\Sigma$ each of whose vertices is a vertex of a convex polytope $\mathcal{P}$ is said to be a special simplex in $\mathcal{P}$ if each facet of $\mathcal{P}$ contains exactly $q-1$ of the vertices of $\Sigma$. It will be proved that there is a special simplex in a compressed polytope $\mathcal{P}$ if (and only if) its toric ring $K[\mathcal{P}]$ is Gorenstein. In consequence it follows that the $h$-vector of a Gorenstein toric ring $K[\mathcal{P}]$ is unimodal if $\mathcal{P}$ is compressed.
\end{abstract}

A compressed polytope [10, p. 337] is an integral convex polytope all of whose "pulling triangulations" are unimodular. (Recall that an integral convex polytope is an convex polytope each of whose vertices has integer coordinates.) A typical example of compressed polytopes is the Birkhoff polytopes [10, Example 2.4 (b)]. Later, in [6], a large class of compressed polytopes including the Birkhoff polytopes is presented. Recently, Seth Sullivant [12] proved a surprising result that the class given in [6] does essentially contain all compressed polytopes. 
Let $\mathcal{P} \subset \mathbb{R}^{n}$ be an integral convex polytope. Let $K$ be a field and $K\left[\mathbf{x}, \mathbf{x}^{-1}, t\right]=$ $K\left[x_{1}, x_{1}^{-1}, \ldots, x_{n}, x_{n}^{-1}, t\right]$ the Laurent polynomial ring in $n+1$ variables over $K$. The toric ring of $\mathcal{P}$ is the subalgebra $K[\mathcal{P}]$ of $K\left[\mathbf{x}, \mathbf{x}^{-1}, t\right]$ which is generated by those monomials $\mathbf{x}^{\mathbf{a}} t=x_{1}^{a_{1}} \cdots x_{n}^{a_{n}} t$ such that $\mathbf{a}=\left(a_{1}, \ldots, a_{n}\right)$ belongs to $\mathcal{P} \cap \mathbb{Z}^{n}$. We will regard $K[\mathcal{P}]$ as a homogeneous algebra [2, p. 147] by setting each $\operatorname{deg} \mathbf{x}^{\mathbf{a}} t=1$ and write $F(K[\mathcal{P}], \lambda)$ for its Hilbert series. One has $F(K[\mathcal{P}], \lambda)=\left(h_{0}+h_{1} \lambda+\cdots+h_{s} \lambda^{s}\right) /(1-\lambda)^{d+1}$, where each $h_{i} \in \mathbb{Z}$ with $h_{s} \neq 0$ and where $d$ is the dimension of $\mathcal{P}$. The sequence $h(K[\mathcal{P}])=\left(h_{0}, h_{1}, \ldots, h_{s}\right)$ is called the $h$-vector of $K[\mathcal{P}]$. If the toric ring $K[\mathcal{P}]$ is normal, then $K[\mathcal{P}]$ is CohenMacaulay. If $K[\mathcal{P}]$ is Cohen-Macaulay, then the $h$-vector of $K[\mathcal{P}]$ is nonnegative, i.e., each $h_{i} \geq 0$. Moreover, if $K[\mathcal{P}]$ is Gorenstein, then the $h$-vector of $K[\mathcal{P}]$ is symmetric, i.e., $h_{i}=h_{s-i}$ for all $i$.

A well-known conjecture is that the $h$-vector $\left(h_{0}, h_{1}, \ldots, h_{s}\right)$ of a Gorenstein toric ring is unimodal, i.e., $h_{0} \leq h_{1} \leq \cdots \leq h_{[s / 2]}$. One of the effective techniques to show that $\left(h_{0}, h_{1}, \ldots, h_{s}\right)$ is unimodal is to find a simplicial complex polytope of dimension $s-1$ whose $h$-vector $[11$, p. 75$]$ coincides with $\left(h_{0}, h_{1}, \ldots, h_{s}\right)$ (Stanley [9]). In fact, Reiner and Welker [8] succeeded in showing that the $h$-vector of a Gorenstein toric ring arising from a finite distributive lattice (see, e.g., [4]) is equal to the $h$-vector of a simplicial convex polytope.

Christos Athanasiadis [1] introduced the concept of a "special simplex" in a convex polytope. Let $\mathcal{P} \subset \mathbb{R}^{n}$ be a convex polytope. A $(q-1)$-simplex $\Sigma$ each of whose vertices is a vertex of $\mathcal{P}$ is said to be a special simplex in $\mathcal{P}$ if each facet (maximal face) of $\mathcal{P}$ contains exactly $q-1$ of the vertices of $\Sigma$. It turns out [1, Theorem 3.5] that if $\mathcal{P}$ is compressed and if there is a special simplex in $\mathcal{P}$, then the $h$-vector of $K[\mathcal{P}]$ is equal to the $h$-vector of a simplicial convex polytope. In particular, if $\mathcal{P}$ is compressed and if there is a special simplex in $\mathcal{P}$, then $K[\mathcal{P}]$ is Gorenstein whose $h$-vector is unimodal. Examples for which [1, Theorem 3.5] can be applied include (i) toric rings of the Birkhoff polytopes ([1, Example 3.1]), (ii) Gorenstein toric rings arising from finite distributive lattices ([1, Example 3.2]), and (iii) Gorenstein toric rings arising from stable polytopes of perfect graphs ([7, Theorem $3.1(\mathrm{~b})]$ ).

In the present paper we prove that there is a special simplex in a compressed polytope $\mathcal{P}$ if (and only if) its toric ring $K[\mathcal{P}]$ is Gorenstein.

Theorem 0.1 Let $\mathcal{P}$ be a compressed polytope. Then there exists a special simplex in $\mathcal{P}$ if (and only if) its toric ring $K[\mathcal{P}]$ is Gorenstein.

Proof. It follows from [12, Theorem 2.4] that every compressed polytope $\mathcal{P}$ is lattice isomorphic to an integral convex polytope of the form $C_{n} \bigcap L$, where $C_{n} \subset \mathbb{R}^{n}$ is the $n$-dimensional unit hypercube and where $L$ is an affine subspace of $\mathbb{R}^{n}$. Without loss of generality, one can assume that $L \bigcap\left(C_{n} \backslash \partial C_{n}\right) \neq \emptyset$, where $\partial C_{n}$ is the boundary of $C_{n}$. In other words, $\operatorname{dim} \mathcal{P}=\operatorname{dim} L$. Let $\mathcal{P}=C_{n} \bigcap L$ with $d=\operatorname{dim} \mathcal{P}$. Thus $L$ is the intersection of $n-d$ hyperplanes in $\mathbb{R}^{n}$, say

$$
\begin{aligned}
& a_{11} x_{1}+\cdots+a_{1 d} x_{d}+x_{d+1}=b_{1} \\
& a_{21} x_{1}+\cdots+a_{2 d} x_{d}+x_{d+2}=b_{2}
\end{aligned}
$$




$$
a_{n-d, 1} x_{1}+\cdots+a_{n-d, d} x_{d}+x_{n}=b_{n-d}
$$

where $a_{i j}, b_{i} \in \mathbb{Q}$ for all $i$ and $j$. Since $\mathcal{P}$ possesses the integer decomposition property [6, p. 2544], its toric ring coincides with the Ehrhart ring [5, p. 97] of $\mathcal{P}$. Hence the criterion [3, Corollary 1.2] can be applied for $K[\mathcal{P}]$.

To state the criterion [3, Corollary 1.2], let $\delta>0$ denote the smallest integer for which $\delta(\mathcal{P} \backslash \partial \mathcal{P}) \bigcap \mathbb{Z}^{n} \neq \emptyset$, where $\delta(\mathcal{P} \backslash \partial \mathcal{P})=\{\delta \alpha: \alpha \in \mathcal{P} \backslash \partial \mathcal{P}\}$, and $\left(c_{1}, \ldots, c_{n}\right) \in$ $\delta(\mathcal{P} \backslash \partial \mathcal{P}) \cap \mathbb{Z}^{n}$. Write $\mathcal{Q} \subset \mathbb{R}^{d}$ for the convex polytope defined by the inequalities

$$
0 \leq x_{i} \leq 1, \quad 1 \leq i \leq d
$$

together with

$$
\begin{gathered}
0 \leq b_{1}-\left(a_{11} x_{1}+\cdots+a_{1 d} x_{d}\right) \leq 1 \\
0 \leq b_{2}-\left(a_{21} x_{1}+\cdots+a_{2 d} x_{d}\right) \leq 1 \\
\cdots \\
0 \leq b_{n-d}-\left(a_{n-d, 1} x_{1}+\cdots+a_{n-d, d} x_{d}\right) \leq 1
\end{gathered}
$$

Then $\mathcal{Q}$ is an integral convex polytope of dimension $d$ with $K[\mathcal{Q}] \cong K[\mathcal{P}]$. Let $\mathcal{Q}^{\sharp}=$ $\delta \mathcal{Q}-\left(c_{1}, \ldots, c_{d}\right)$. Then $\mathcal{Q}^{\sharp}$ is an integral convex polytope of dimension $d$ and the origin of $\mathbb{R}^{d}$ belongs to the interior of $\mathcal{Q}^{\sharp}$. By using [3, Corollary 1.2] the toric ring $K[\mathcal{Q}]$ is Gorenstein if and only if the equation of the supporting hyperplane of each facet of $\mathcal{Q}^{\sharp}$ is of the form $q_{1} x_{1}+\cdots+q_{d} x_{d}=1$ with each $q_{j} \in \mathbb{Z}$.

Claim. Suppose that $K[\mathcal{Q}]$ is Gorenstein. Then, for each $1 \leq i \leq n$, one has $c_{i}=\delta-1$ (resp. $c_{i}=1$ ) if the hyperplane in $\mathbb{R}^{n}$ defined by the equation $x_{i}=1$ (resp. $x_{i}=0$ ) is a supporting hyperplane of a facet of $\mathcal{P}$.

Proof of Claim. Let $1 \leq i \leq d$. If the equation $x_{i}=1$ (resp. $x_{i}=0$ ) defines a facet of $\mathcal{P}$, then the equation $x_{i}+c_{i}=\delta$ (resp. $x_{i}+c_{i}=0$ ) defines a facet of $\mathcal{Q}^{\sharp}$. Since $0 \leq c_{i} \leq \delta$, one has $c_{i}=\delta-1$ (resp. $\left.c_{i}=1\right)$, as desired.

Let $1 \leq i \leq n-d$. If the equation $x_{d+i}=1$ defines a facet of $\mathcal{P}$, then the equation

$$
a_{i 1}\left(x_{1}+c_{1}\right)+\cdots+a_{i d}\left(x_{d}+c_{d}\right)=\delta\left(b_{i}-1\right)
$$

defines a facet of $\mathcal{Q}^{\sharp}$. Since $a_{i 1} c_{1}+\cdots+a_{i d} c_{d}+c_{d+i}=\delta b_{i}$, the equation

$$
a_{i 1} x_{1}+\cdots+a_{i d} x_{d}=c_{d+i}-\delta
$$

defines a facet of $\mathcal{Q}^{\sharp}$. We write the equation (1) of the form

$$
(p / q)\left(a_{i 1}^{\prime} x_{1}+\cdots+a_{i d}^{\prime} x_{d}\right)=c_{d+i}-\delta
$$

where $a_{i 1}^{\prime}, \ldots, a_{i d}^{\prime}$ are integers which are relatively prime, and where $p$ and $q>0$ are integers which are relatively prime. Then $q\left(c_{d+i}-\delta\right) / p= \pm 1$. Hence $q=1$. Thus each 
$a_{i j} \in \mathbb{Z}$ is divided by $p$. We write the equation $a_{i 1} x_{1}+\cdots+a_{i d} x_{d}+x_{d+i}=b_{i}$ of the form $p\left(a_{i 1}^{\prime} x_{1}+\cdots+a_{i d}^{\prime} x_{d}\right)+x_{d+i}=b_{i}$. Since $L \bigcap\left(C_{n} \backslash \partial C_{n}\right) \neq \emptyset$, there is a vertex $\left(v_{1}, \ldots, v_{n}\right)$ of $\mathcal{P}=C_{n} \cap L$ with $v_{d+i}=0$. Thus $b_{i} \in \mathbb{Z}$ is divided by $p$, say, $b_{i}=p b_{i}^{\prime}$ with $b_{i}^{\prime} \in \mathbb{Z}$. Let $\left(v_{1}, \ldots, v_{n}\right)$ be a vertex of $\mathcal{P}$ with $v_{d+i}=1$. However, unless $p= \pm 1$, such the vertex cannot lie on the hyperplane defined by the equation $p\left(a_{i 1}^{\prime} x_{1}+\cdots+a_{i d}^{\prime} x_{d}\right)+x_{d+i}=p b_{i}^{\prime}$. Thus $p= \pm 1$. Since $c_{d+i}-\delta=p$ and $c_{d+i} \leq \delta$, one has $p=-1$ and $c_{d+i}=\delta-1$, as desired. On the other hand, modify the above technique slightly, and one has $c_{d+i}=1$ if the hyperplane in $\mathbb{R}^{n}$ defined by the equation $x_{d+i}=0$.

Now, we proceed to the final step of our proof of Theorem 0.1. Since $\left(c_{1}, \ldots, c_{n}\right)$ belongs to $\delta(\mathcal{P} \backslash \partial \mathcal{P}) \cap \mathbb{Z}^{n}$, there exists $\delta$ vertices $\mathbf{v}_{1}, \ldots, \mathbf{v}_{\delta}$ of $\mathcal{P}$ with $\left(c_{1}, \ldots, c_{n}\right)=$ $\mathbf{v}_{1}+\cdots+\mathbf{v}_{\delta}$. Write $\Sigma$ for the convex hull of $\left\{\mathbf{v}_{1}, \ldots, \mathbf{v}_{\delta}\right\}$. Our work is to show that $\Sigma$ is a special simplex in $\mathcal{P}$. Let a facet $\mathcal{F}$ of $\mathcal{P}$ be defined by the equation $x_{i}=1$ (resp. $\left.x_{i}=0\right)$. Then $c_{i}=\delta-1$ (resp. $\left.c_{i}=1\right)$. Since each vertex of $\mathcal{P}$ is a $(0,1)$-vector, exactly $\delta-1$ vertices of $\mathbf{v}_{1}, \ldots, \mathbf{v}_{\delta}$ lie on $\mathcal{F}$. Finally, to see why $\Sigma$ is a $(\delta-1)$-simplex, suppose that, say, $\mathbf{v}_{\delta}$ belongs to the convex hull of $\left\{\mathbf{v}_{1}, \ldots, \mathbf{v}_{\delta-1}\right\}$ and that $\mathbf{v}_{\delta}$ does not lie on a facet $\mathcal{G}$ of $\mathcal{P}$. Then all of $\mathbf{v}_{1}, \ldots, \mathbf{v}_{\delta-1}$ must belong to $\mathcal{G}$. Hence $\Sigma \subset \mathcal{G}$. Thus $\mathbf{v}_{n} \in \mathcal{G}$, which contradicts $\mathbf{v}_{n} \notin \mathcal{G}$.

Q. E. D.

By virtue of $[1$, Theorem 3.5] together with Theorem 0.1 it follows that

Corollary 0.2 Let $\mathcal{P}$ be a compressed polytope and suppose that the toric ring $K[\mathcal{P}]$ is Gorenstein. Then the h-vector of $K[\mathcal{P}]$ is unimodal.

\section{References}

[1] C. A. Athanasiadis, Ehrhart polynomials, simplicial polytopes, magic squares and a conjecture of Stanley, J. Reine Angew. Math. 583 (2005), 163 - 174.

[2] W. Bruns and J. Herzog, "Cohen-Macaulay Rings," Revised Ed., Cambridge Studies in Advanced Mathematics 39, Canbridge University Press, Cambridge, 1998.

[3] E. De Negri and T. Hibi, Gorenstein algebras of Veronese type, J. Algebra 193 (1997), $629-639$.

[4] T. Hibi, Distributive lattices, affine semigroup rings and algebras with straightening laws, in "Commutative Algebra and Combinatorics" (M. Nagata and H. Matsumura, Eds.), Advanced Studies in Pure Math., Volume 11, North-Holland, Amsterdam, 1987, pp. $93-109$.

[5] T. Hibi, "Algebraic Combinatorics on Convex Polytopes," Carslaw, Glebe, N.S.W., Australia, 1992.

[6] H. Ohsugi and T. Hibi, Convex polytopes all of whose reverse lexicographic initial ideals are squarefree, Proc. Amer. Math. Soc. 129 (2001), 2541 - 2546. 
[7] H. Ohsugi and T. Hibi, Special simplices and Gorenstein toric rings, J. Combin. Theory, Ser. A, in press.

[8] V. Reiner and V. Welker, On the Charney-Davis and Neggers-Stanley conjectures, J. Combin. Theory, Ser. A 109 (2005), 247 - 280.

[9] R. P. Stanley, The number of faces of a simplicial convex polytope, Advances in Math. 35 (1980), $236-238$.

[10] R. P. Stanley, Decompositions of rational convex polytopes, Annals of Discrete Math. 6 (1980), $333-342$.

[11] R. P. Stanley, "Combinatorics and Commutative Algebra," Second Ed., Progress in Mathematics 41, Birkhäuser, Boston / Basel / Stuttgart, 1996.

[12] S. Sullivant, Compressed polytopes and statistical disclosure limitation, arXiv:math.CO/0412535, 2004. 